\title{
Content-based load balancing of tasks using task clustering for cost optimisation in cloud computing environment
}

\section{Kaushik Sekaran*}

Department of Computer Science and Engineering,

Mahatma Gandhi Institute of Technology,

Hyderabad, 500075, India

Email:kaushik.vit@gmail.com

Email: skaushik_cse@mgit.ac.in

*Corresponding author

\section{P. Venkata Krishna}

Department of Computer Science and Engineering,

Sri Padmavati Mahila Visvavidyalayam,

Tirupati, India

Email: dr.krishna@ieee.org

\begin{abstract}
Cloud computing is the recent mantra for all the techies and internet users all around the world. The power of cloud computing is enormous as it provides big services in an optimal cost as well as in a reliable manner. Load balancing of tasks in the cloud server is an important issue to be addressed. In this paper, we propose a task clustering algorithm to minimise the load across the cloud servers through content-based load balancing of tasks using task clustering methods and cost reduction method for optimal energy consumption at all the cloud data centre heads. The results analysed in our paper are better when compared with existing content-based load balancing models. Our approach clearly represents the achievement of optimal load balancing of tasks with respect to upload bandwidth utilisation, minimal latency and some other quality of service (QoS) metrics.
\end{abstract}

Keywords: cloud computing; load balancing; tasks clustering; cost reduction; energy consumption; QoS; quality of service; metrics.

Reference to this paper should be made as follows: Sekaran, K. and Krishna, P.V. (2022) 'Content-based load balancing of tasks using task clustering for cost optimisation in cloud computing environment', Int. J. Advanced Intelligence Paradigms, Vol. 21, Nos. 1/2, pp.1-17.

Biographical notes: Kaushik Sekaran received his Bachelor of Technology in Computer Science and Engineering from the Sastra University, Thanjavur in year 2005. He then pursued postgraduate studies and received his Master of Engineering in Computer Science and Engineering from the Mepco Schlenk Engineering College, Affiliated to Anna University, Chennai in year 2008. He then pursued research studies and received his $\mathrm{PhD}$ degree from the VIT University, Vellore in year 2015. He is currently an Associate Professor in the School of Computer Science and Engineering at VIT University, Vellore, India. His current research interests include in the areas of cloud computing, bio-inspired computing, big data analytics, internet of things (IoT) and security issues in peer-to-peer networks. 
P. Venkata Krishna received his BTech in Electronics and Communication Engineering from the Sri Venkateswara University, Tirupathi, India. He then pursued MTech in Computer Science and Engineering from the Regional Engineering College, Calicut, India and $\mathrm{PhD}$ degree from the VIT University, Vellore, India. He is currently a Professor in Department of Computer Science and Engineering, Sri Padmavati Mahila Visvavidyalayam, Government State Level University, Tirupati, India. His research interests include mobile, wireless systems, grid computing and cloud computing.

\section{Introduction}

The cloud load balancing is a challenging job in the cloud computing environment. As the cloud users are continuously raising various kinds of requests such as cloud services request and many other cloud-based requests. There are many cloud services as suggested by Quarati et al. (2014) are available in cloud computing. One of the cloud service models is infrastructure as a service (IaaS), in that model, the actual infrastructures such as servers, hardware disks, etc. are shared via internet for many number of users over the cloud.

Many researchers said about many services in the cloud. Most cloud level services are meant to do the jobs according to cloud service level agreement (SLA). In the SLA negotiation between the cloud user and the cloud service providers, many policies have been discussed and decided. After that mutually both parties may agree to share the resources between the various cloud servers across the globe. The cloud users' start sending the requests to the private cloud such as Amazon EC2 or Azure cloud platform, they have to wait and get the resources within the particular time frame. Due to low bandwidth ratio in some segments across the internet, the contents will be delivered to the users in a delayed manner. In order to reduce this kind of delay, all the contents that are transmitted over the internet via cloud servers should be segregated according to the type of the content. The tasks that are assigned to the cloud server may clustered according to their contents such as video streaming content as said by Savas et al. (2012), audio content and other files content. Many content-based load balancing scenario is available over the internet.

Cloud load balancing scenario is clearly demonstrated by Google's cloud compute model. Cloud load balancing is works with an efficient module called cloud load balancer, a hardware unit which routing the traffic according to all the incoming HTTP and HTTPS traffic. This router works only for few protocols. Some of the algorithms that sit over this router can make many magic such as routing the traffic according to the content of the incoming tasks and clustering the tasks according to their file type. We are proposing an algorithm named task clustering algorithm for doing this kind of load balancing. 
Figure 1 Load balancing scenario in cloud (see online version for colours)

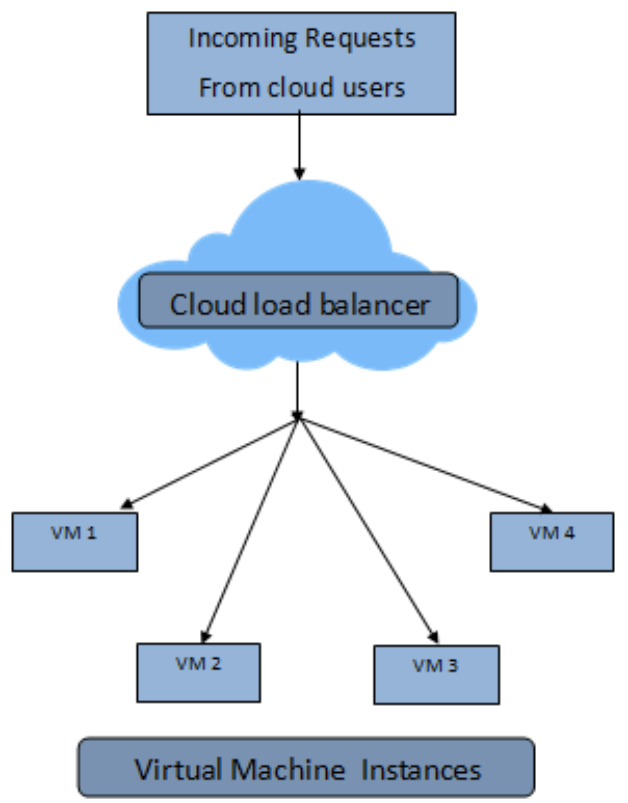

Figure 1 clearly represents the load balancing for all incoming user requests by cloud load balancer. It distributes all the incoming network traffic across multiple virtual machine instances. Cloud load balancers manage online traffic by distributing workloads across multiple servers and resources - automatically or on demand. They maximise your workload performance and help prevent overload.

\subsection{Benefits of load balancing of tasks}

The cloud load balancing in the cloud computing environment provides the following advantages:

- $\quad$ application scaling

- heavy traffic analysis and minimisation

- balance loads across multiple cloud servers

- route traffic to the closest virtual machine.

The work in this paper, content configuration load balancing, uses cloud load balancer with HTTP load balancing to distribute traffic to different VM instances based on the incoming requests. This particular scenario is depicted in Figure 2. The video content files are distributed to server and further sent to the respective (virtual machines) VMs, i.e., VM1, VM2, etc. As the same way other content files are also have their own distribution among the respective VMs. 
Figure 2 Content-based load balancing (see online version for colours)

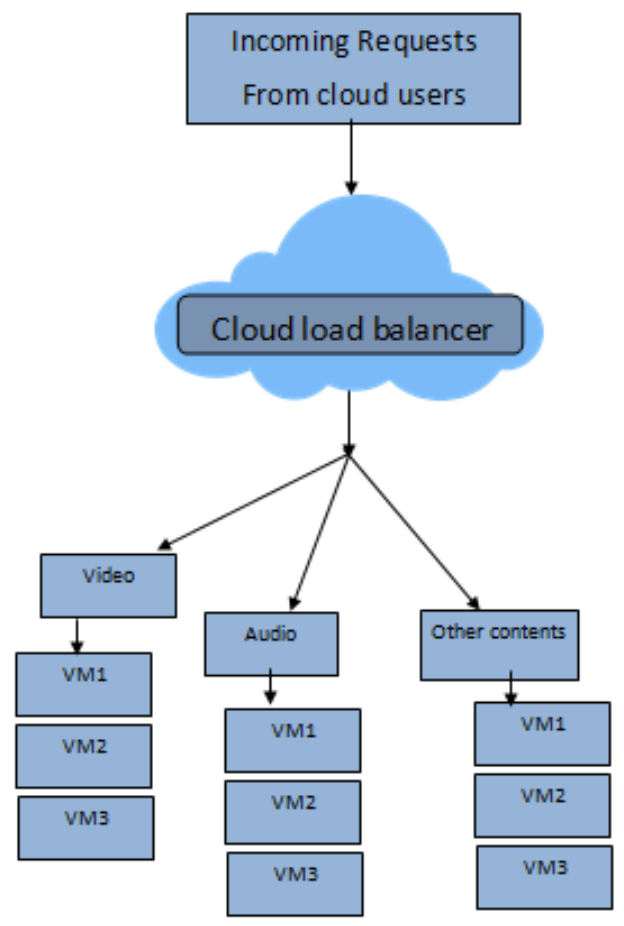

\section{Related work}

Some of the earlier content-based load balancing of tasks had many methods to provide optimal load balancing. Moltchanov (2011) mentioned about some of the quality of service (Qos) and discussed about the problems related to the quality of video streaming in peer to peer systems, reasons for not incorporating the mechanism to deliver a quality media. The task clustering method is the upcoming scenario that we are proposing as a new idea in this paper.

There are some video streaming methods as proposed as a novel solution for streaming multi-view video as suggested by Savas et al. (2012) over P2P overlay networks by providing techniques and methods for generating scalable chunks and methods that achieve the possibility of high quality video streaming. In a survey on load balancing by Al Nuaimi et al (2012) in cloud computing clearly describes, as there are some algorithms and challenges, researchers investigated the different algorithms proposed to solve the problems of balancing load and scheduling task with latest approaches. Ramzan et al. (2012) described the discussion of existing video streaming mechanisms in P2P systems considering the parameters of efficiency based on tree-based and mesh-based systems. Mohamed et al. (2013) said about the files downloading can be done much fast with dynamic load-balancing in the cloud with a dual direction technique. Some data scientists have introduced an efficient and fast concurrent technique that downloads large files from replicated data centres of cloud. Balancing load in data centre is a crucial part where few methods have been introduced about threshold-based dynamic 
compare and balancing algorithm. Sahu et al (2013) designed scheduling the resources based on the system usage and performance this approach can reduce service cost reduction in cloud industry. In recent years, there are some algorithm for load balancing is designed which is inspired by the honey bee behaviour by Dhinesh Babu and Krishna (2013) that priorities the task with the amount of waiting time of the task in the queue is minimal, the results shown is effective than the existing algorithm on load balancing. Also, few algorithms has introduced cloud brokering system introduced by Quarati et al. (2014), that is based on the gateway toolkit, this proposed system provides advantages to customers and cloud brokers in the hybrid cloud.

A novel design of hierarchical cloudlet-based storage architecture designed by Duro et al. (2014) for mobile devices has been introduced for smart computing innovations and researches in video streaming. There are some proposal about cloud scheduler as suggested by Pacini et al. (2014) which is based on ant colony optimisation that studies the performance metrics and total VMs created with scheduling scenarios, the results balancing of load in cloud is effective than the existing scheduler. In some research articles, the author proposed a new load balancing method that propose a resource grouping strategy as proposed by Shen et al. (2014) and balancing the load among the groups, by the technique network congestion identifying is more possible and efficient, also there is the need in streaming media that has been presented as a distributed sub stream scheduling as said by $\mathrm{Hu}$ et al. (2014) and distributed scheme with block live streaming which shows the result has better efficiency in video streaming. Number theory towards energy efficient scheduling as said by Chen et al. (2015) which it dynamically exploits proactive scheduling methods, they proposed three strategies and four typical baseline algorithms for scheduling. A novel P2P infrastructure designed by Fabian and Feldhaus (2014) for organised private data query in the larger peer to peer networks of smart appliances is one of the base concepts for peer to peer video streaming strategies.

Energy efficient for data centres are more considered which have proposed a load balancing algorithm the is based on the adjustment on the server speed and routing of workload derived by Ko et al. (2014) in the server that optimises load in data centres results shows the algorithm proposed is effective in data centres. In some work proposed for virtual machine by Dong et al. (2014) and optimised virtualisation of technology have been using the application widely in cloud data centres, they aim to optimise the network traffic that reduces the congestion in network. The results shows maximum link utilisation is reduced to $20 \%$ in IaaS in cloud computing. Few works in the field of cloud resource management Manvi et al. (2014) have surveyed on provided techniques which are resource mapping, adaption, provisioning. Earlier P2P streaming methods (Chen et al., 2014) have developed a mathematical model for peer to peer voice on demands to trace the evolution of peer, revealing the origin cause towards loading of server in population decay. Video pricing mechanism by Cong et al. (2014) has been proposed that allocates video instances and pricing of each video instance with the auction algorithm on low bid to determine payment and allocation of video instances. Recovering video stream losses through the internet (Dandoush et al., 2014) has been introduced as an algorithm by two way mechanisms to recover lost data in peer to peer systems. The result analysis shows the cost of overhead, complexity and bandwidth in the systems. Video streaming for mobile devices (Jin et al., 2015) with interaction that is derived based on the game model have been presented by some authors. The big cloud model as proposed by Sekaran and Krishna (2016) considered to be one of the best ways to solve the cloud 
computing load balancing issues in a simpler way. The model proposed is a striker strategy that leads the system to enhance performance of the system. Many application oriented issues (Detti et al., 2015) have been raised for designing an application in peer to peer that streams video live with content encoded bit streams that exploit main functionalities they measured the performance and results shows effectiveness. Dynamic Load balancing (Susila et al., 2014) using firefly load balancing algorithm to balance the load in numerous partitions in cloud computing has been introduced with the logic results that shows better performance in terms of computational time, task migration and load arrival ratio. Also, heterogeneous cloud model as presented by Behal and Kumar (2014), the researchers has some good analysis on performance of load balancing algorithms in cloud environment in terms of response time using efficient load balancing policies on it.

\subsection{Identification of research gaps and proposed models}

First of all, to point out some existing content-based load balancing scenarios and research gaps in this area, we need to understand about Google cloud platform (https://cloud.google.com/compute/docs/load-balancing/http/content-based-example) and Amazon web services (AWS) (https://aws.amazon.com/cloudfront/), in which the HTTP load balancing has been done for the video and audio contents for the load balancing methods. They have implemented the HTTP/HTTPS connections by configuring the VM instances in different zones. The major issues focussed in Google cloud platform are about DNS-based cloud IP address matching. In that the DNS resolver has been taking much response time from the each and every cloud data centres. In our approach the response time has been greatly reduced by is in the specific content-based load balancing routine in the query forwarding.

\section{Proposed work}

Cloud user has several needs for storage, compute and networking in cloud environment. The contents may differ according to the cloud server availability. The tasks submitted by the cloud user through the cloud domain are processed in the Task clustering server. Figure 3 representing the architectural framework for content-based load balancing in cloud. The hierarchy is followed from the cloud user to cloud server via task clustering server, which is clustering the task according to the incoming content from the cloud users. Our proposed task clustering algorithm explains about the task clustering method that is given in the next section.

\subsection{Content-based task clustering using content filtering cloud server}

Cloud user's tasks are clustered and classified into groups and processed in the respective VMs. Each VMs has unique server configuration based on the type of task it is clustered. Allocation of tasks is done at the clustering server side and tasks are scheduled to the VMs. Cloud has remote clustered VMs and their remote addresses are stored in the index of the clustering server. Each task has different content in it and assigned to the individual VMs in order to optimise the load. By content-based load balancing, the overall cloud server throughput is high and hence the task clustering performance is increased. The content filtering cloud server is also called as dominant cloud server. The 
dominant cloud server takes active role in segregating the contents after vibrant analysis of the incoming clustered file such as clustered video file embedded with hash values through hash function $\mathrm{H}(\mathrm{x})$. Similarly the other contents are also clustered using content filtering cloud server to give original content to the cloud user as represented in Figure 4.

Figure 3 Architectural framework for content-based load balancing in cloud (see online version for colours)

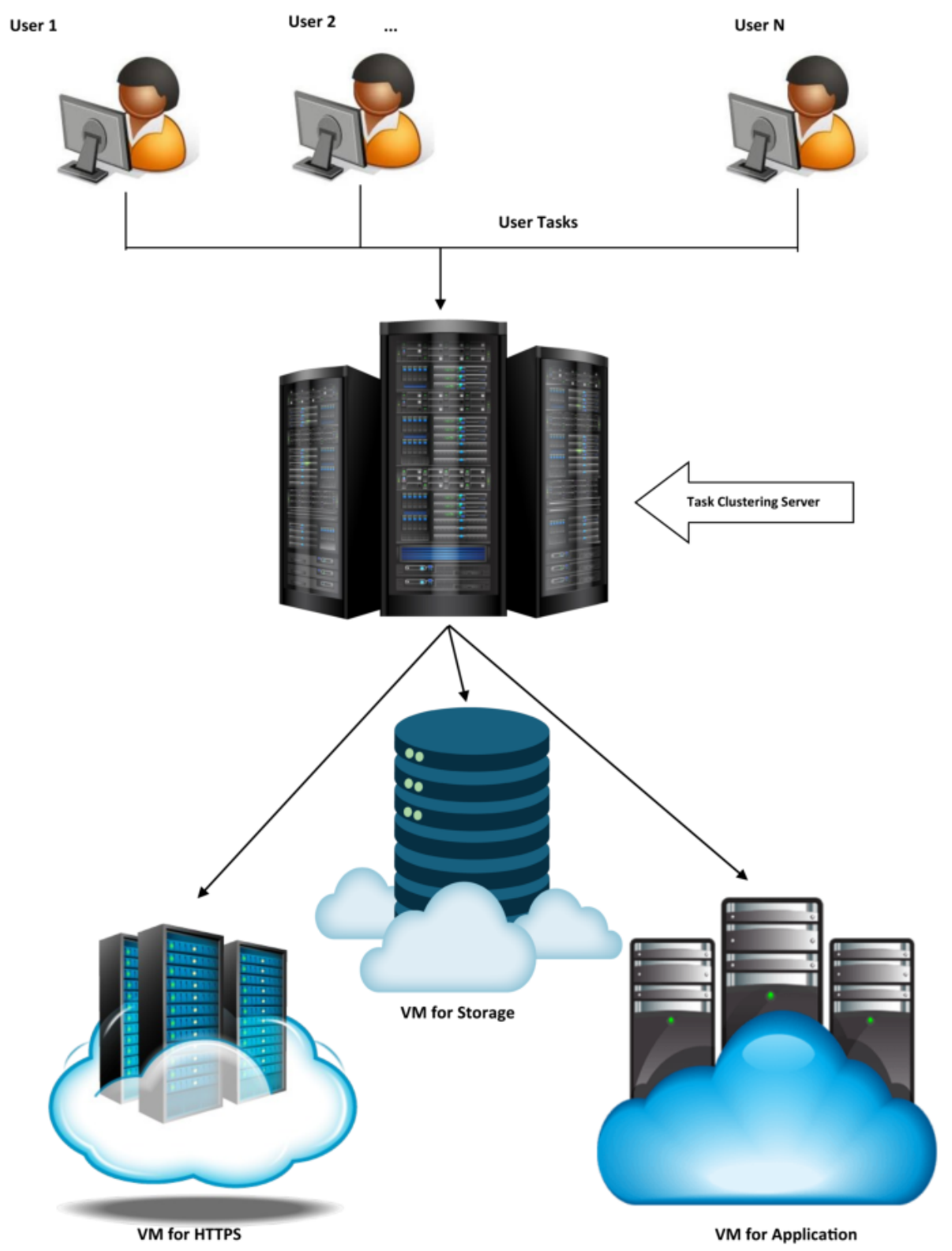


Figure 4 Content filtering cloud server for load balancing among the different contents (see online version for colours)

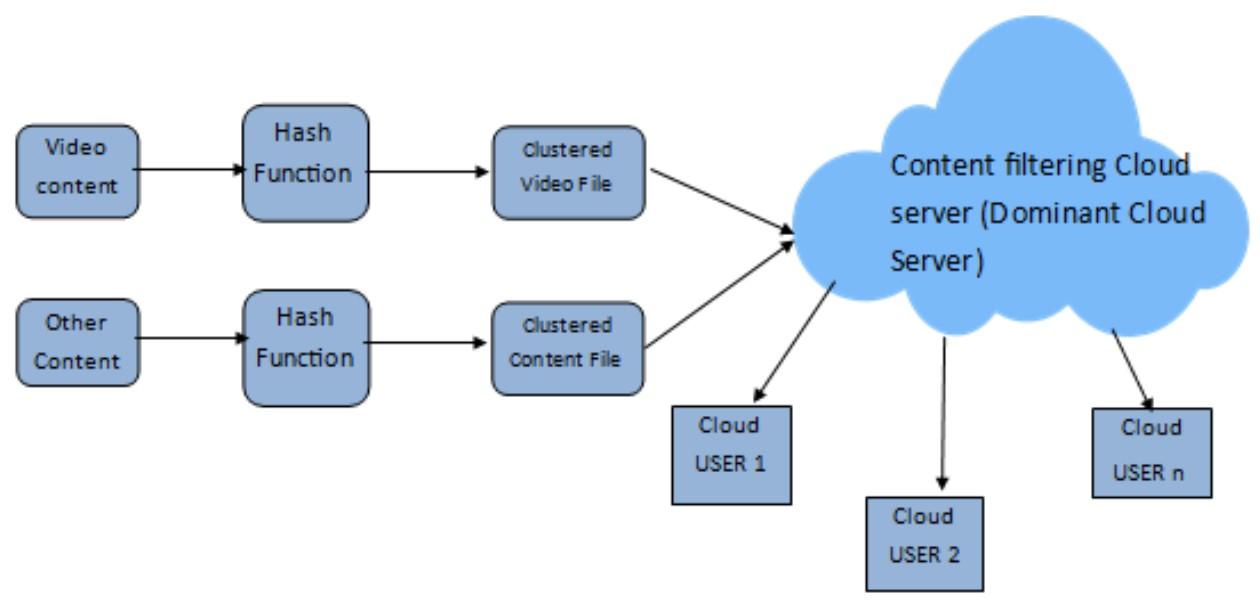

\section{Analytical model}

\subsection{Notations used for task clustering algorithm for load balancing of tasks}

In the given in Table 1, it is easy understand some of the standard notations used in this proposed model. Content-based load balancing of tasks is one of the key research areas in the multimedia cloud computing model. The content of every tasks are clustered using task clustering server. Every tasks and the content in it have been assigned with an index, i.e., for referring in the server, each content should have its own unique identity for its lookup service. For that, we are introducing distributed hash table (DHT) concept, to map every content with the respective server.

Table 1 Notations used for task clustering algorithm for load balancing of tasks

\begin{tabular}{lc}
\hline Symbol & Meaning \\
\hline$D H T_{n}$ & Distributed hash table \\
$V . D H T_{n}$ & Video streaming task's index \\
$C S n$ & Cloud servers \\
$C U n$ & Cloud users \\
$V M$ & Virtual machine \\
$R T$ & Response time \\
$B W u$ & Bandwidth utilisation \\
\hline
\end{tabular}

The role of DHT is very useful in assigning a key value to the contents. DHT usages are huge as like increased bandwidth utilisation, low level latency in searching the content and increased hard disk capacity to provide a better file-sharing service among nearest cloud servers. Assume there are several contents in a task clustering cloud server. Then, a proper DHT mechanism should be there to organise all the tasks in a linear way. Here our assumption is, if there are ' $n$ ' number of DHT is assigned for every video-based 
incoming contents into the task clustering server, then $D H T_{1}$ to $\mathrm{D} D H T_{m}$ belongs to a common index called $D H T_{n}$, that is given in the equation (1).

$$
\sum D H T_{n} \epsilon\left\{D H T_{1}+D H T_{2}+\cdots D H T_{m}\right\}
$$

Also, some DHT formation has to be designed more secure against some malicious online attacks. And the DHT must be assigned with some strong key pair to avoid the third party attacks. Already DHTs are dealt with many distributed systems applications such as load balancing, data integrity and performance related QoS issues.

Figure 5 Process map for clustering of tasks in cloud

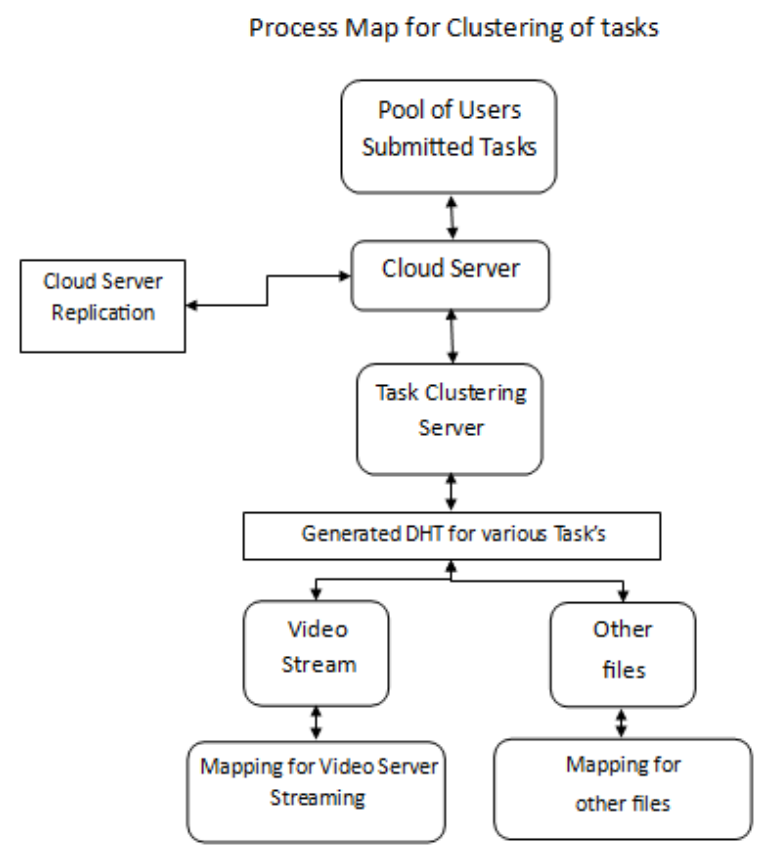

The process map given in Figure 5 represents the flow of the proposed work.

Steps involved in the process map:

Step 1 Initially, the pool of users submitting their tasks to the cloud server.

Step 2 Then there is a cloud server replica that takes every backup of files that is stored in the cloud server.

Step 3 According to the incoming tasks, contents are distributed to the respective servers by generating and assigning the DHTs to it.

Step4 If there are video stream files, they are sent to the video cloud server for further process such as video streaming into the website.

Step 5 Similarly for other contents, the same procedure will be continuing. 


\section{Task clustering algorithm for content-based load balancing of tasks}

Figure 6 represents the life cycle of multimedia cloud systems. It composed of various stages of processes such as procurement, storing in Multimedia cloud, processing, distribution and presentation. Always the multimedia content starts their life cycle from procurement such as raw data of any video or audio content. Then the particular media content will be sent for processing such as video processing, image processing or audio synchronisation processing and all other processing stuffs.

Figure 6 Multimedia cloud life cycle (see online version for colours)

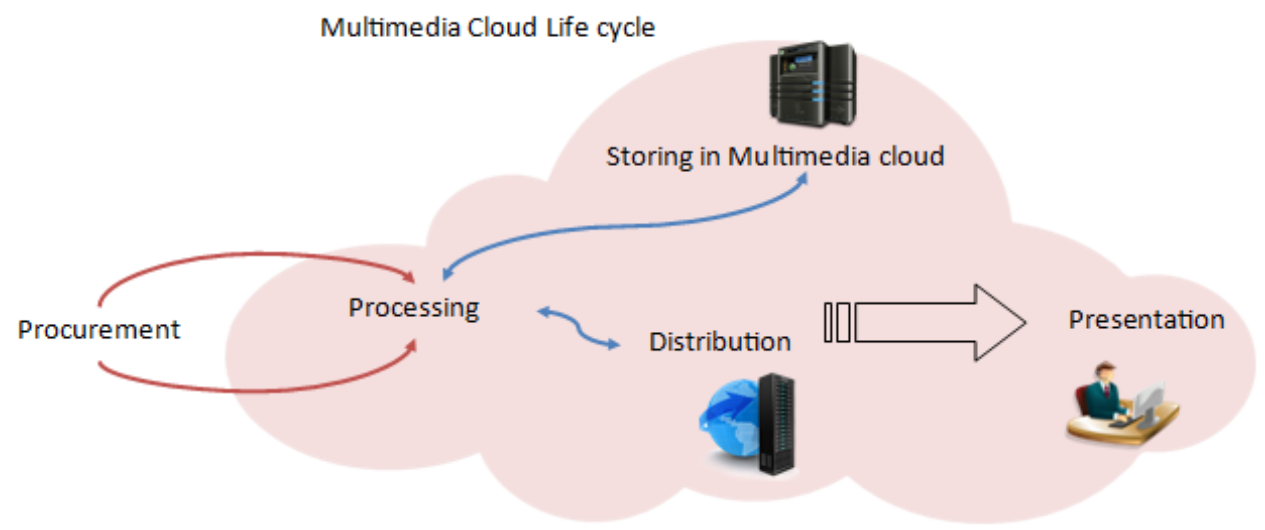

After the processing stage, it's sent to the cloud data centre for the storage of the multimedia content files. Then it is broadcasted to all the cloud data centres for the application purpose. So only we are able to see the video files in the presentation stage such as video streaming through youtube.com kind of websites.

Cloud user's tasks are clustered and classified into groups and processed in the respective VMs. Each VMs has unique server configuration based on the type of task it is clustered. Allocation of tasks is done at the clustering server side and tasks are scheduled to the VMs.

Figure 7 depicts the flow of work in accessing the physical resources in multimedia cloud systems such as cloud data centre by public with delivering cloud services for multimedia such as video streaming service and multimedia storage services. After allotting a particular service to the cloud user, then the content-based load balancer module will check and filter the contents and it will send it to the cloud data centre according to its availability update in the cloud status table. Through proper virtual machine instances concept the user task has been executed.

We are proposing task clustering algorithm to minimise the latency in the cloud server. Figure 8 clearly representing its flow of process. Firstly, there are many users who need immediate response from the cloud server sending their queries continuously from any of the public cloud model such as Google cloud bus. 
Figure 7 Cloud services for multimedia content (see online version for colours)

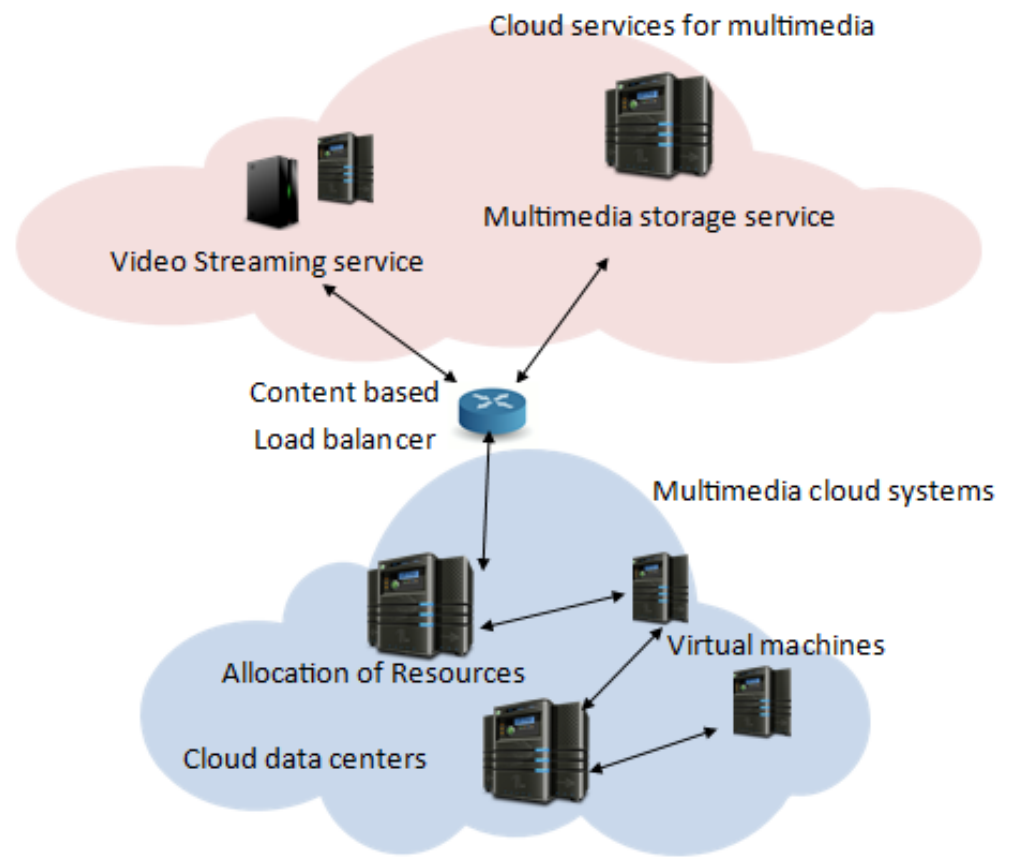

By upload of tasks into the task clustering server, the tasks are actually splits according to the content in it. DHTs are assigned to each task. For example, if it is a video steaming task, then $V . D H T_{1} \ldots$ to $V . D H T_{n}$ will be assigned according to the incoming tasks. Similarly this method will be continuing for other related contents also. Our proposed algorithm explains clearly about this process using java-based methods.

Algorithm 1 Task clustering procedure

Procedure AssignContentsToVMs(contentList)

Begin

$$
\begin{aligned}
& \mathrm{DHT}=0 \\
& \text { vmIndex }=0 \\
& \text { while }(\text { ContentList.size }()>0) \\
& \text { Content }=\text { ContentList.getNextContent }() \\
& \mathrm{vm}=\operatorname{getVMsList}(\mathrm{vmIndex}) \\
& \text { vm.scheduleContentToVM(Content) } \\
& \text { DHT.assignContenttoDHT(DHTList) } \\
& \text { totalVMs }=\text { getVMsList().size() } \\
& \text { vmIndex }=\operatorname{Mod}(\text { vmIndex }+1 \text {,totalVMs }) \\
& \text { DHT }=\operatorname{Mod}(\text { DHTList }+1 \text {,totalDHTs }) \\
& \text { ContentList.remove(Content) }
\end{aligned}
$$

end while 
Figure 8 Assigning DHT for incoming contents in cloud server (see online version for colours)

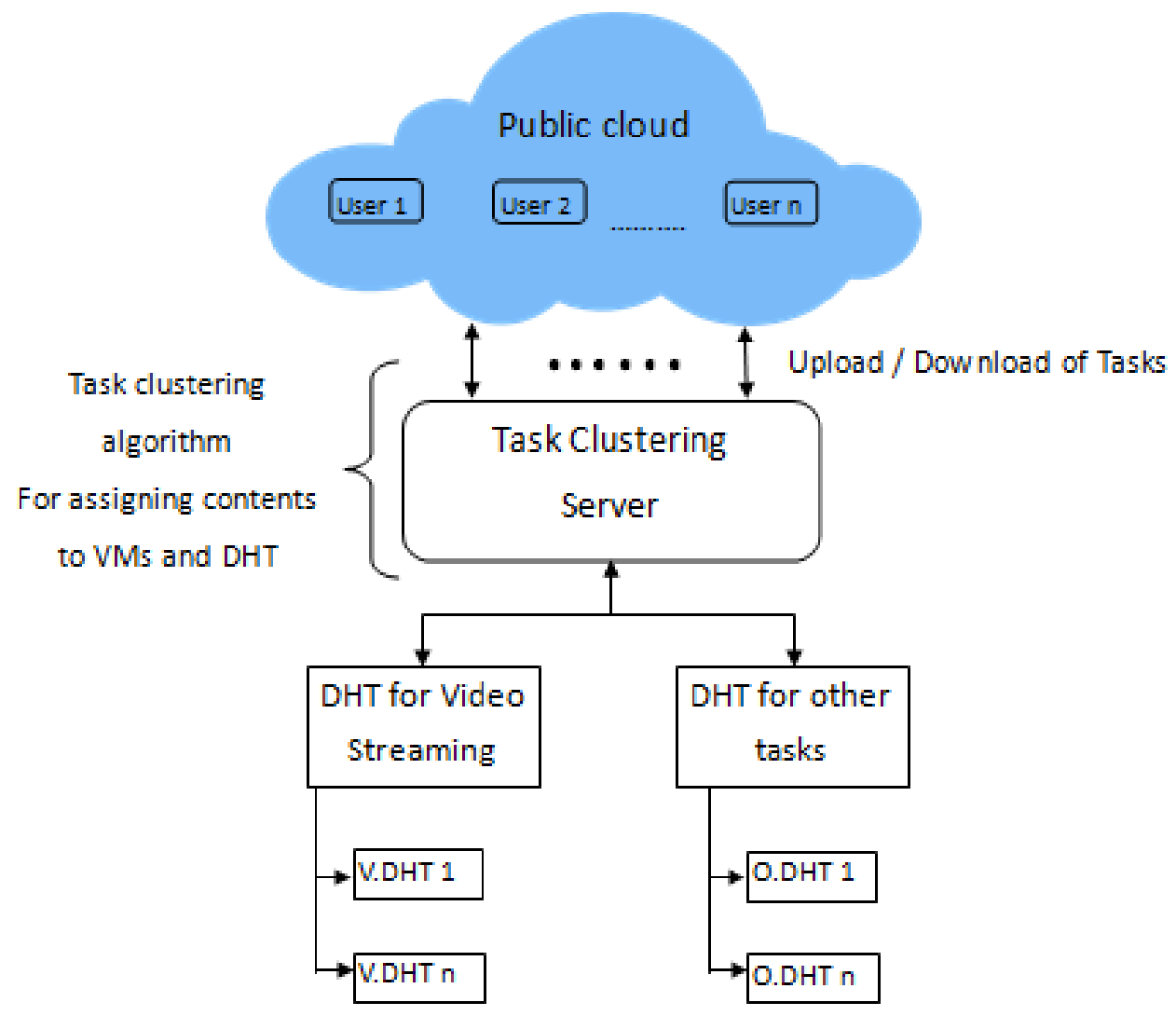

This algorithm depicts the overall content assignment to the respective VMs in the cloud computing environment. The algorithm uses two different lists, the first one is about various kinds of requests (denoted as contents) from the cloud user and the other is about DHT mapping with all the incoming contents. This specified algorithm having repeated procedural steps for ContentListand then, over getNextContent() method that is used for getting response from Task clustering Server in a (FirstInFirstOut )FIFO manner. And every content is sent to the respective content VMs in a Round-robin method. The method getVMsList(vmIndex) is used for task completion in the VM. These steps are repeated until the ContentList is denoted for NULL value and all tasks have been submitted for completion in the Task clustering ServerVM.

\subsection{Content configuration problem to reduce cost in cloud data centres}

Content like video files can be streamed across various countries from different cloud data centres. Content configuration problem may happen in cloud data centres. This problem can be solved only by analysing the cost per data centre for the entire content deliveries. Assume if there are 100 cloud data centres located across the country and then there might be at least 1,000 times greater ratio of the users who dependent on the particular cloud data centres in the respective region (Sekaran and Krishna, 2017). The 
cost factor is an important and essential parameter, which has to be calculated in order to analyse about the resource mapping for the users. In order to reduce the cost among many clusters in the cloud data centre, firstly the initial cost has to be calculated.

Initial cost $\left(C_{I}\right)$ is expressed by adding data centre ownership cost $\left(C_{O}\right)$ and existing cluster cost $\left(C_{C}\right)$ that is given in equation (2).

$$
C_{I}=C_{O}+C_{C}
$$

Also, after calculating the $\left(C_{I}\right)$, then it is easy to analyse the data centres overall costs $\left(C_{D O V}\right)$ by adding the $C_{I}$, cost for energy consumption of independent nodes $C_{E}$, cost for task migration among cloud data centres VMs CTM and cost for workload calculation $\left(C_{W C}\right)$ given in equation (3).

$$
C_{D O V}=C_{I}+C_{E}+C_{T M}+C_{W C}
$$

\subsection{Experimental model for analysing cost reduction}

In Cloud computing environments the cost analysing is done by creating comprehensive model in any tool to understand about the actual cost reduction in the cloud data centres. We have used dynamic cloudsim tool to configure and analyse our results. Assume there are four data centre heads located in four different location and they have costs such as initial cost and energy consumption of independent nodes $C_{E}$. We are introducing an even analysis model table and graph to understand this scenario. From Table 2 we could clearly understand about the given scenario.

Figure 9 Cost calculation in data centre heads (see online version for colours)

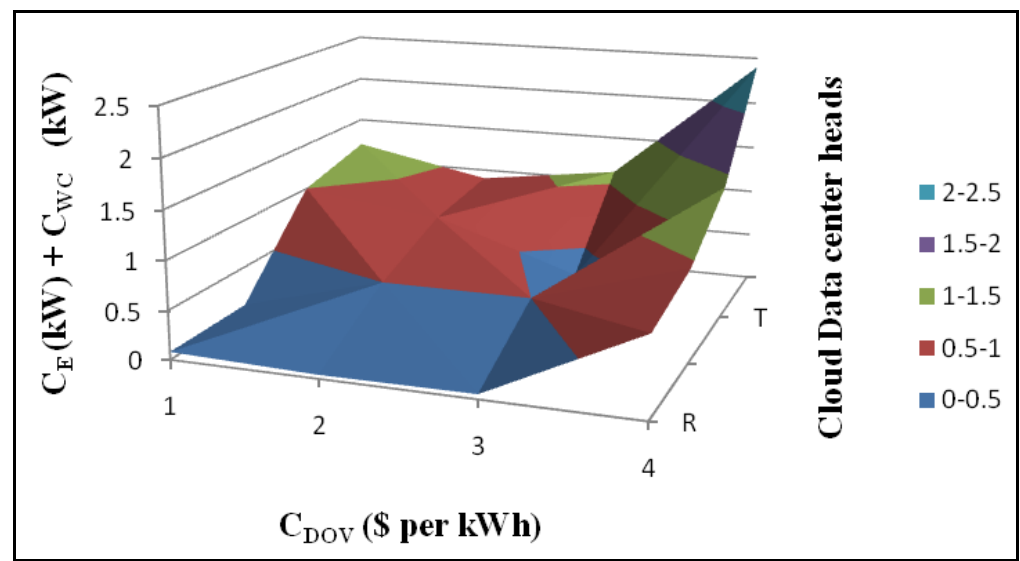

$$
C_{E}(k W)=C_{C}+C_{I D}
$$

$C_{E}(k W)$ is calculated by the equation (4), where $C_{I D}$ is the energy consumption cost in the idle machines and $\left(C_{C}\right)$ existing cluster cost. Figure 9 depicts the overall $C_{D O V}$ cost in kilowatt per hour for all the four cloud data centre heads. In that, the data centre $U$, having more energy spent due to many modules such as energy consumption by individual cloud data centre as well as idle machines in the cloud data centre represented 
as $C_{E}(\mathrm{~kW})$ and the workload calculation for every cloud data centre $C_{W C}(\mathrm{~kW})$ is more when compared to the other cloud data centre heads.

Table 2 Cost calculation in data centre heads considering various cost parameters

\begin{tabular}{|c|c|c|c|c|}
\hline \multirow{4}{*}{$\begin{array}{l}\text { Cloud data } \\
\text { centre heads }\end{array}$} & \multicolumn{4}{|c|}{ Cost calculation in data centre heads } \\
\hline & \multicolumn{4}{|c|}{ Various cost parameters } \\
\hline & \multirow[t]{2}{*}{$C_{D O V}(\$$ per $k W h)$} & \multicolumn{2}{|c|}{$C_{E}(\mathrm{~kW})$} & \multirow[t]{2}{*}{$C_{W C}(\mathrm{~kW})$} \\
\hline & & $C_{C}$ & $C_{I D}$ & \\
\hline $\mathrm{R}$ & 0.08 & 0.04 & 0.05 & 0.81 \\
\hline $\mathrm{S}$ & 0.09 & 0.5 & 0.5 & 0.96 \\
\hline $\mathrm{T}$ & 1 & 0.8 & 0.3 & 1.4 \\
\hline $\mathrm{U}$ & 1.2 & 0.9 & 1.1 & 2.4 \\
\hline
\end{tabular}

\section{Experimental setup}

There are various contents of tasks are coming into the cloud server, in which the load is equally analysed and the content-based filtering is done in the task clustering cloud server, the upload bandwidth utilisation and latency in streaming the video contents between the VMs are calculated using CloudSim simulation. Figure 10 represents the decreased video latency time (ms) versus number of VMs. The result proves that, if we increase the number of VMs in each cloud server, the processing could be simpler than the existing method which saves the video latency time, hence there is about $20 \%$ faster retrieval time in retrieving the video data.

Figure 10 Number of VMs vs. video latency time (ms)

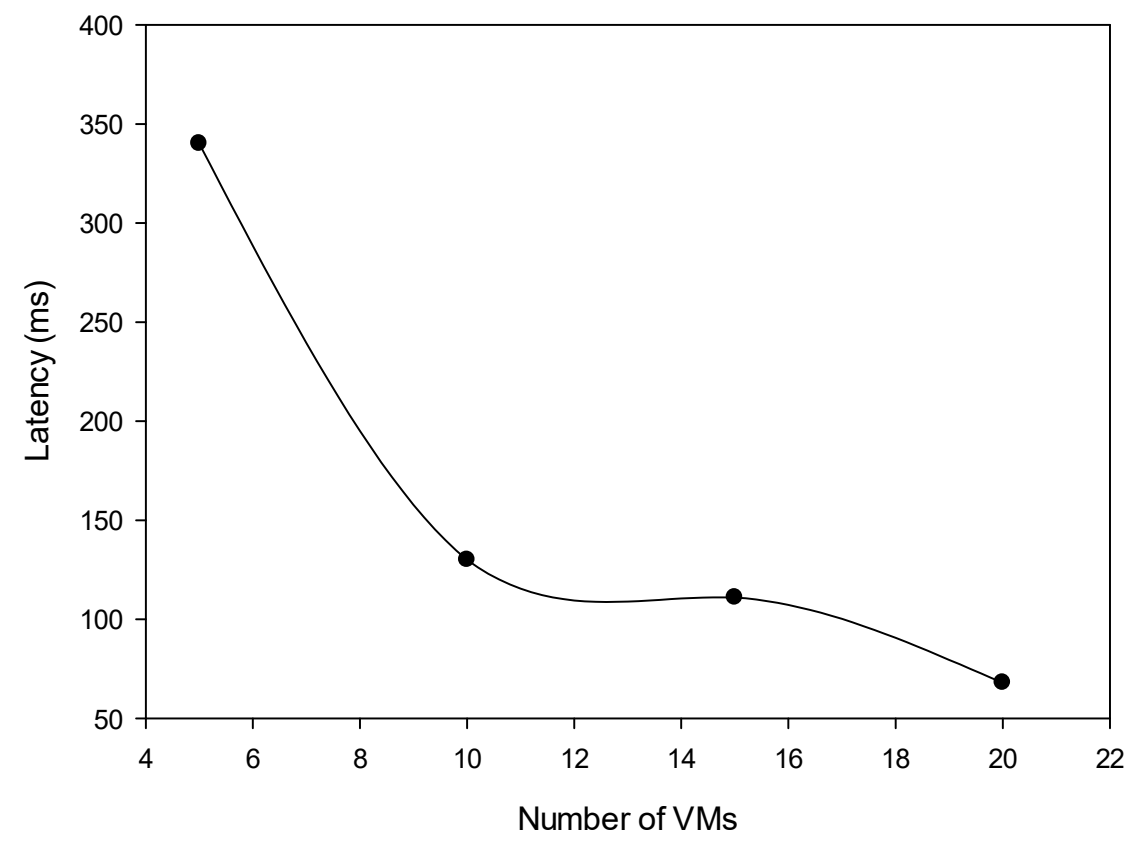


Table 3 Video upload bandwidth utilisation calculation using task clustering algorithm for video tasks

\begin{tabular}{lcc}
\hline No. of tasks & No. of VMs & Upload bandwidth utilisation(mbps) \\
\hline 10 & 5 & 1.3 \\
20 & 10 & 4 \\
30 & 15 & 5.6 \\
40 & 20 & 8 \\
50 & 25 & 12.48 \\
\hline
\end{tabular}

Figure 11 Video upload bandwidth utilisation (mbps) graph (see online version for colours)

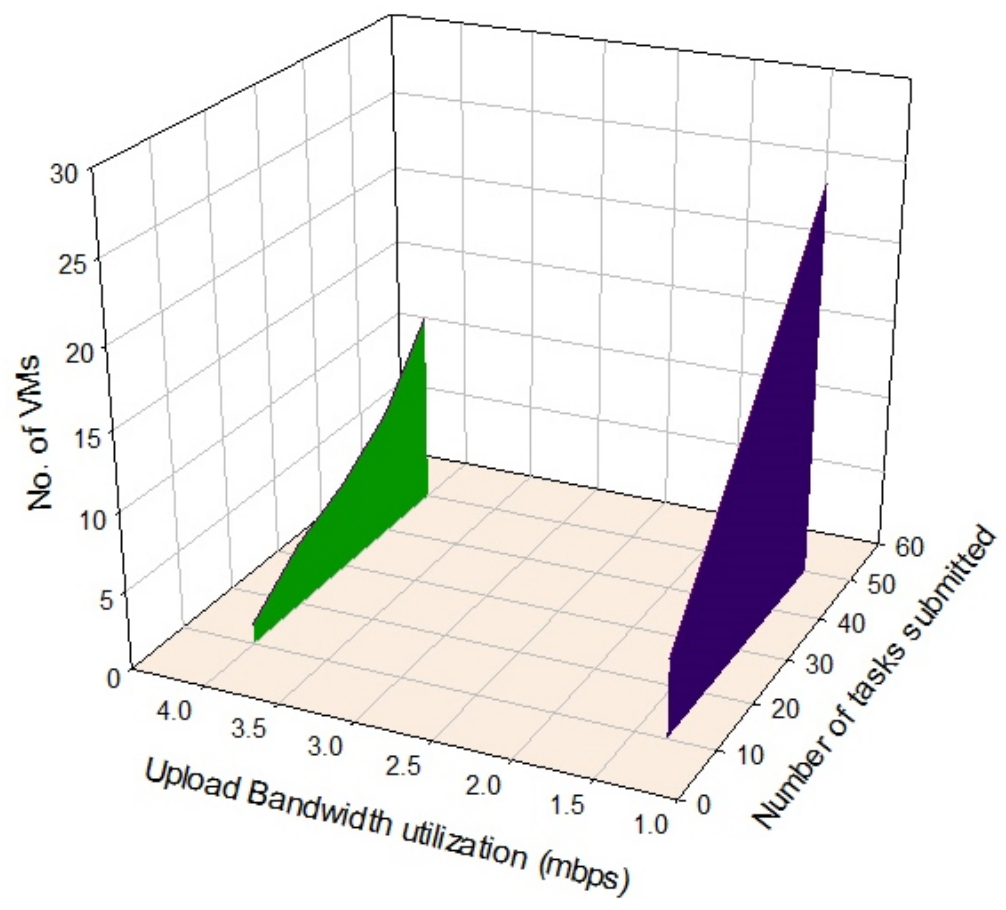

From Table 3, we can clearly understand about upload video bandwidth utilisation by different cloud servers. The video upload bandwidth utilisation of the cloud servers for various workload distributions is analysed by creating 200 cloud servers and each server is assumed in single core having up to 4,000mips (millions instructions per second) with 8GB RAM and 1TB storage. The users submit the tasks for provided that of 200 cloud server VMs. Every VM is assumed to have an equal and distributed load in the cloud server. On Net beans IDE with CloudSim, this setup is tested to find the optimal standard deviation values. Hence we can clearly understand that the proposed algorithm is considered to be utilising less bandwidth among multiple cloud servers by using the good content-based load balancing technique. Figure 11 represents the video upload bandwidth utilisation (mbps) graph, which consists of three axes. In that X-axis represents the number of tasks submitted by the users and the Y-axis represents the number of VMs and $\mathrm{Z}$-axis represents bandwidth utilisation by the servers in mbps (megabits per second) unit. 


\section{Conclusions and future work}

The content-based load balancing of tasks method benefits the digital society in multiple ways. Firstly, this method reduces the video latency time in the virtual machine instances across various cloud servers that helps in better video streaming in popular socio internet websites like Youtube.com. And the video upload bandwidth utilisation (mbps) is better when compared to the lot of existing methodologies.

In this paper, we have analysed and validated our work for content-based load balancing of tasks in cloud computing environment. This approach helps in balancing the load in multiple cloud servers by investigating QoS metrics such as video tasks upload bandwidth utilisation and latency. Also, in the proposed task clustering algorithm, it is clearly seen that there is a reduced latency in the streaming of videos. Our result shows good improvement in video tasks upload bandwidth utilisation among cloud servers. We plan to extend this algorithm for private cloud storage mechanisms to balance the tasks based on the contents stored and to decrease the buffering speed of the video further more in near future. The computational cost model in cloud can be taken into account for the future study for the better energy consumption in the cloud data centres.

\section{References}

Al Nuaimi, K., Mohamed, N., Al Nuaimi, M. and Al-Jaroodi, J. (2012) 'A survey of load balancing in cloud computing: challenges and algorithms', IEEE Second Symposium on Network Cloud Computing and Applications.

Behal, V. and Kumar, A. (2014) Cloud Computing: Performance Analysis of Load Balancing Algorithms in Cloud Heterogeneous Environment, IEEE, 978-1-4799-4236-7/14.

Chen, F., Li, H. and Liu, J. (2014) 'Popularity decays in peer-to-peer VoD systems: impact, model and design implications', Science Direct Computer Networks, Vol. 60, pp.75-87.

Chen, H., Zhu, X., Guo, H., Zhu, J., Qin, X. and Wu, J. (2015) 'Towards energy-efficient scheduling for real-time tasks under uncertain cloud computing environment', Science Direct, The Journal of Systems and Software, Vol. 99, pp.20-35.

Cong, X., Shuang, K. Su, S. Yang, F.C. and Zi, L. (2014) 'LBAS: an effective pricing mechanism towards video migration in cloud-assisted VoD system', Science Direct Computer Networks, Vol. 64, pp.15-25.

Dandoush, A., Alouf, S. and Nain, P. (2014) 'Lifetime and availability of data stored on a P2P system: evaluation of redundancy and recovery schemes', Science Direct Computer Networks, Vol. 64, pp.243-260.

Detti, A., Ricci, B. and Blefari-Melazzi, N. (2015) 'Mobile peer-to-peer video streaming over information-centric networks, Computer Networks, Vol. 81, pp.272-288, DOI: http://dx.doi.org/10.1016/j.comnet.2015.02.018

Dhinesh Babu, L.D. and Krishna, P.V. (2013) 'Honey bee behavior inspired load balancing of tasks in cloud computing environments', Sci. Verse Science Direct Applied Soft Computing Vol. 13, No. 5, pp.2292-2303.

Dong, J-.K., Wang, H-Bo., Li, Y-Y. and Cheng, S-D. (2014) 'Virtual machine placement optimizing to improve network performance in cloud data centers', Science Direct The Journal of China Universities of Posts and Telecommunications, June, Vol. 21, No. 3, pp.62-70.

Duro, F.R., Blas, J.G., Higuero, D. Perez, O. and Carretero, J. (2014) 'CoSMiC: a hierarchical cloudlet-based storage architecture for mobile clouds', Science Direct Simulation Modelling Practice and Theory, Vol. 50, pp.3-19. 
Fabian, B. and Feldhaus, T. (2014) 'Privacy-preserving data infrastructure for smart home appliances based on the Octopus DHT', Science Direct Computers in Industry, Vol. 65, pp.1147-1160.

Hu, C., Chen, M., Xing, C. and Zhang, G. (2014) 'Exploring the optimal substream scheduling and distribution mechanism for data-driven P2P media streaming', Science Direct Computer Communications, Vol. 44, pp.14-25.

Jin, X. and Kwok, Y-K. (2015) 'Coercion builds cooperation in dynamic and heterogeneous P2P live streaming networks', Science Direct Computer Networks, Vol. 81, pp.1-18.

Ko, Y.M. and Cho, Y. (2014) 'A distributed speed scaling and load balancing algorithm for energy efficient data centers', Science Direct Performance Evaluation, Vol. 79, pp.120-133.

Manvi, S.S. and Shyam, G.K. (2014) 'Resource management for infrastructure as a service (IaaS) in cloud computing: a survey', Science Direct Journal of Network and Computer Applications, Vol. 41, pp.424-440.

Mohamed, N., Al-Jaroodi, Jameela and Eid, A. (2013) 'A dual-direction technique for fast file downloads with dynamic load balancing in the Cloud', Sci. Verse Science Direct Journal of Network and Computer Applications, Vol. 36, No. 4, pp.1116-1130.

Moltchanov, D. (2011) 'Service quality in P2P streaming systems', Elsevier Computer Science Review, pp.5319-340

Pacini, E., Mateos, C. and Garino, C. (2014) 'Balancing throughput and response time in online scientific clouds via ant colony optimization', Science Direct Advances in Engineering Software, Vol. 84, pp.31-47.

Quarati, A. Clematis, A., D’Agostino, D. (2014) 'Delivering cloud services with QoS requirements: business opportunities, architectural solutions and energy-saving aspects', Science Direct Future Generation Computer Systems, Vol. 55, pp.403-427.

Ramzan, N., Park, H. and Izquierdo, E. (2012) 'Video streaming over P2P networks: Challenges and opportunities', Sci. Verse Science Direct Signal Processing: Image Communication, Vol. 27, No. 5, pp.401-411.

Savas, S.S., Goktuğ Gurler, C., Murat Tekalp, A., Ekmekcioglu, E., Worrall, S. and Kondoz, A. (2012) 'Adaptive streaming of multi-view video over P2P networks', Sci. Verse Science Direct Image Communication, Vol. 27, No. 5, pp.522-531.

Sekaran, K. and Krishna, P.V. (2016) 'Big cloud: a hybrid cloud model for secure data storage through cloud space', International Journal of Advanced Intelligence Paradigms, Vol. 8, No. 2, pp.229-241.

Sekaran, K. and Krishna, P.V. (2017) 'Cross region load balancing of tasks using region-based rerouting of loads in cloud computing environment', International Journal of Advanced Intelligence Paradigms, Vol. 9, Nos. 5-6, pp.589-603.

Shen, X-J., Liu, L., Zha, Z-J., Gu, P-Y., Jiang, Z-Q., Chen, J-M. and Panneerselvam, J. (2014) 'Achieving dynamic load balancing through mobile agents in small world P2P networks', Science Direct Computer Networks, Vol. 75, Part A, pp.134-148.

Susila, N., Chandramathi, S. and Kishore, R. (2014) 'A fuzzy-based firefly algorithm for dynamic load balancing in cloud computing environment', Journal of Emerging Technologies in Web Intelligence, November, Vol. 6, No. 4, pp.435-440.

Yatendra, S., Pateriya, R.K. and Gupta, R.K. (2013) 'Cloud server optimization with load balancing and green computing techniques using dynamic compare and balance algorithm', 5th International Conference on Computational Intelligence and Communication Networks, pp.527-531.

\section{Websites}

https://cloud.google.com/compute/docs/load-balancing/http/content-based-example.

https://aws.amazon.com/cloudfront/. 